\title{
ИННОВАЦИИ. СИСТЕМНЫЕ ОСНОВЫ
}

\author{
С.В. Семенов, к.т.н., с.н.с. \\ (НИИ "Центрпрограмлсистем", пр. 50 лет Октября, За, г. Тверь, 170024, Россия, \\ semenov_58@mail.ru)
}

Аннотация. Проблемы формирования хороших инновационных проектов обусловлены тем, что заинтересованные в них стороны, отстаивая свои интересы, не понимают друг друга, а главное - не понимают сути собственно инновации и инновационной деятельности. С системной точки зрения инновационный проект существенно отличается от других видов проектов, поэтому традиционные методики их оценки здесь не применимы.

Для понимания устройства инновационного проекта нужно рассмотреть его на атомарном уровне, вникнуть в механизмы формирования сложных информационных структур проекта из отдельных элементов, сформулировать требующиеся и вполне достаточные условия появления хороших упакованных проектов. Это необходимо для правильного написания проекта инноватором и возможности принятия решений о его поддержке заинтересованными сторонами.

В статье рассматриваются вопросы формулирования «элементарных частиц» инновационного проекта для всех заинтересованных сторон, внутренних (умение, желание, возможность) и внешних (новизна, реализуемость и выгодность) свойств этих «атомов». Сформулированы условия притяжения «атомов» через свойства ближней среды (предметная область, математические модели, информационные технологии), а также условия упакованности проекта через учет состояния внешней среды и самого инноватора (его предпринимательские способности, нормативная база, инновационная инфраструктура).

Предложен подход к оценке инновационных проектов на основе математического аппарата теории фракталов, теории полезности и методов функционально-стоимостного анализа. Показана особая роль информационных технологий в процессе создания инновационного информационного пространства.

Ключевые слова: инновачия, инновачионная модель, инновационный проект, атом инновации, информационные технологии.

\section{Общие положения}

Переход на инновационную модель развития требует глубокого понимания таких понятий, как инновация [1] и инновационная деятельность [2]. Важно также разобраться в том, как устроены инновации и инновационная деятельность на системном уровне, который подразумевает описание инноваций и инновационной деятельности как на макро- (уровень политики государства), так и на микроуровне (атомарный уровень).

Понимание устройства и законов инновационного пространства позволит целенаправленно формировать инновационные процессы и политику инновационной экономики.

Как известно, на макроуровне модель инновационной деятельности представляется как взаимодействие трех рынков: рынка идей, рынка товаров и услуг, рынка капитала (рис. 1).

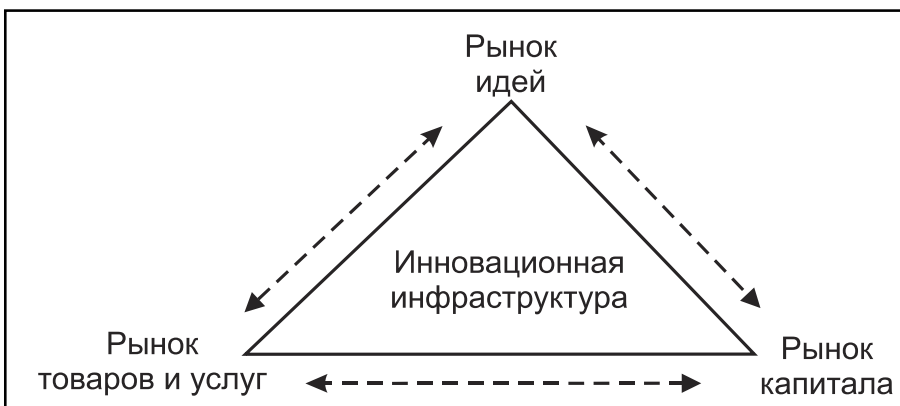

Рис. 1. Обобщенная модель инновационной инфраструктуры

По сути рынок идей - это рынок интеллектуальной собственности и иных результатов интеллектуальной деятельности, рынок капитала - финансовая система, работающая в инвестиционном направлении, рынок товаров и услуг - совокупность рынков производителей и потребителей.

В отличие от традиционной модели экономики [1], основанной на спросе, инновационная экономика строится на достижениях науки и техники, а также потребности в повсеместных изменениях. Конкурентоспособность товаров, предприятий и видов деятельности обусловлена знаниями, источником которых являются все участники производственно-экономических отношений. Совокупность предложений по изменениям, новым видам продуктов и услуг в виде интеллектуальной собственности и составляет новый тип рынка - рынок идей. Именно интеллектуальная собственность, а не товар является основным объектом купли-продажи в инновационной экономике. 
Потребности в изменениях повсеместны и безграничны. Это и новые товары, и услуги, и повышение эффективности какой-либо деятельности, производительности труда на производстве, конкурентоспособности, и потребности в улучшении качества и условий жизни.

В традиционной модели экономики [1] всегда была некая сторона, которая знала, что и как нужно делать. Это и потребители, спрос которых постоянно изучался, и заказчики, составлявшие технические задания. В инновационной экономике такой «знающей» стороны нет - есть намерение что-то изменить либо идея некоторого продукта или услуги. Заранее оценить результаты предлагаемых изменений или определить судьбу будущего продукта невозможно. Но без этих изменений и новых продуктов невозможно ни конкурировать на рынке, ни обеспечить эффективность какой-либо деятельности. Поэтому необходимо создавать условия и правила игры, при которых возможна реализация идей и изменений для всех участников производственно-экономических отношений.

Прежде всего нужно разобраться в системных основах инновационного механизма, чтобы затем целенаправленно выстраивать правила игры и инновационную инфраструктуру, поддерживающую инновационную деятельность [2].

\section{Общая теория систем}

Для построения инновационной модели развития обратимся к общей теории систем. Система в самом общем виде представляется как концепт, субстрат и структура.

Субстрат - это те простейшие структуры или образования, которые остаются устойчивыми и неизменными при любых преобразованиях вещи и обусловливают ее конкретные свойства (например атомы при химических реакциях) [3]. Другими словами, это элементы, из которых будет состоять система.

Структура (или строение) - внутреннее устройство чего-либо, связанное с категориями целого и его частей. Выявление связей, изучение взаимодействия и соподчиненности составных частей различных по своей природе объектов позволяют выявлять аналогии в их организации и изучать структуры абстрактно, без связи с реальными объектами. Другими словами, это связи между элементами системы.

Концепт - системообразующее свойство, то, ради чего создается система, смысл ее существования и основные правила ее функционирования.

В инновационной модели элементами системы являются люди, а также инновационные объекты и субъекты. Люди разделяются на тех, у кого возникают идеи изменить продукт и услугу или создать новые, и тех, кто принимает решение о приобретении и использовании инновационных продуктов и услуг. Субъекты - это элементы инновационной инфраструктуры и внешней среды, в которой происходит движение инноваций [1] от идеи до конечного продукта. Объекты - собственно инновации, постоянно меняющие свою форму представления на протяжении всего жизненного цикла.

Принципиальным элементом в этой системе является именно человек как источник и проводник инновационных идей и изменений.

Зарождение идей и изменений происходит под воздействием многих факторов:

- окружающий мир (экономика, производство, быт);

- виртуальный мир (мир цифровой информации);

- видимые или осознанные несовершенства (понимание того, что может быть лучше);

- идеи, приходящие в голову как озарение.

Накоплен немалый опыт по стимулированию научной и изобретательской мысли, например ТРИЗ [4], эвристические методы и т.п., но это отдельный вопрос. В данной статье рассматриваются условия появления идеи и ее движение по инновационному циклу жизни, а также необходимые условия существования инновационной среды.

\section{Атом инновации}

Мозг человека и есть тот микромир, где рождаются атомы инновационной модели развития. Рассмот-

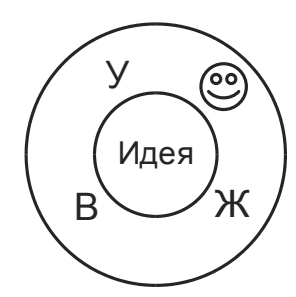

Рис. 2. Атом инновационной модели рим, как устроены эти атомы, являющиеся наименьшими элементами модели (рис. 2).

Инновационная деятельность складывается из деятельности всех заинтересованных сторон. У каждой из них есть свой наименьший элемент деятельности (атом). Атом инноватора отличается от атомов других заинтересованных сторон. Но атом инициатора инновации является главным в инновационной деятельности. Поэтому именно ему будет уделено основное внимание в данной статье.

Ядро атома - это некая идея. Символом :) будем обозначать обязательное присутствие человека в данном элементе модели. 
Если в атоме инноватора идея - это то, что описывает инноватор, то в атоме, например, инвестора идея - это то, что оценивается с точки зрения возможности инвестирования.

Вокруг ядра находится некий информационно-человеческий потенциал в виде элементарных частиц: У - умение, Ж - желание, В - возможность.

Умение - это способность выполнять какие-то действия, общий уровень знаний и навыков, способность понимать и формулировать проблемы и решения, выбирать выразительные средства, производить расчеты, строить модели и т.п.

Желание - мотивированность к началу действий: признание, деньги, внутреннее удовлетворение.

Возможность - условия, позволяющие начать действия: возможность формализации идеи (показать заинтересованной стороне, убедить, зафиксировать результат интеллектуальной деятельности, оценить, изобразить выразительными средствами, возможность быть понятым), использования подходящих правил игры, объектов инфраструктуры, наличие заинтересованных сторон.

Обозначим эту тройку элементарных частиц как УЖВ. Этот потенциал возможных изменений может быть как большим, так и маленьким.

\section{Описание внешней среды атома инноваций}

Внешняя среда (не только инновационная) для развития инновации складывается из многих влияющих на нее факторов. На рисунке 3 представлена модель этих факторов. Часть внешней среды, имеющей отношение к инновационной деятельности, составляет инновационную среду.

В модели каждая окружность представляет собой некоторую сферу чьих-то интересов в области инноваций. В совокупности эти сферы и составляют инновационную среду, в которой реализуется деятельность по выращиванию инновации. Силы и правила, действующие в этих сферах, влияют на каждый инновационный атом. Но степень влияния проявляется в зависимости от близости к центру модели (к атому инновации). Как будет показано далее, выращивание из атома молекулы, из нее - полноценного проекта, а из него - инновационного продукта происходит путем постоянной детализации инновационной идеи и постоянного учета факторов внешней среды. По мере продвижения от центра к краю модели (рис. 3) степень влияния удаленных факторов среды на инновацию возрастает.

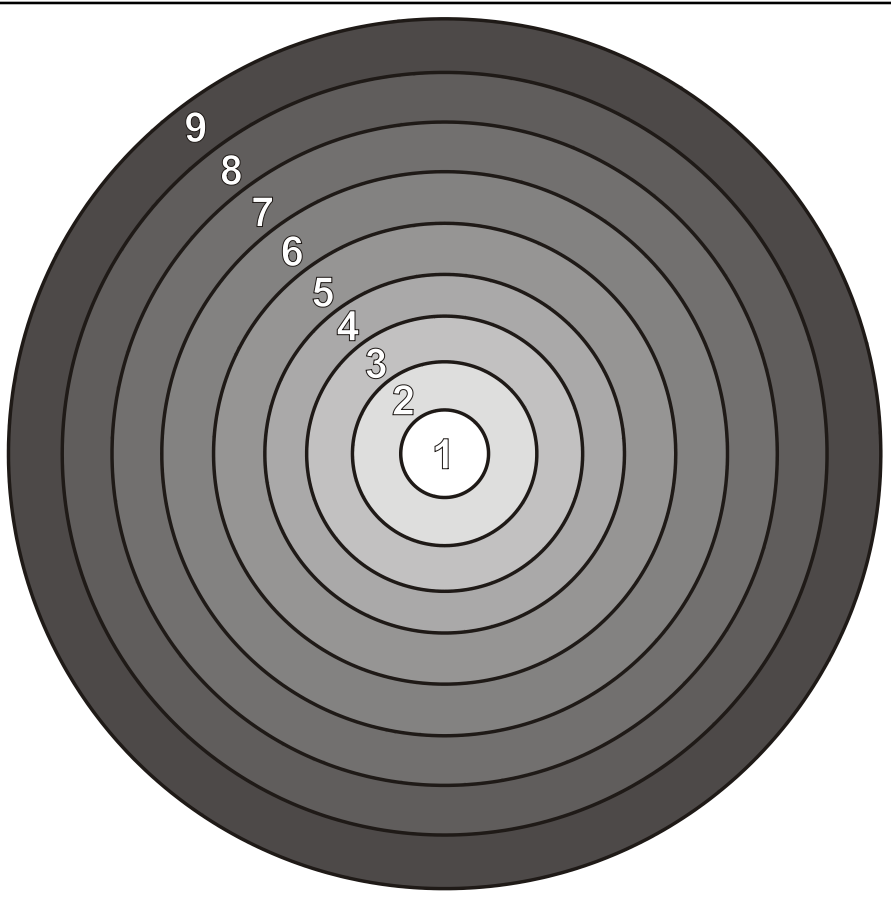

Рис. 3. Модель внешней (инновационной) среды для инновачии:

1 - атом инновации, 2 - сообщество инноваторов и изобретателей, 3 - инновачионная инфраструктура, 4 - сообщество инвесторов, 5 - организации сферы науки, образования и проектирования, 6- промышленные предприятия и предприятия сферы услуг, 7 - конечные потребители инновационных товаров и услуг, 8 - политика государства в области инновационной экономики, 9 -мировая экономика и мировые научно-технические достижения 
Позиция 1 условно представляет атом инновации как один из возможных центров кристаллизации будущей инновации. «Прорастание» инновации происходит под воздействием ближних и дальних сил сфер инновационной среды. При всем многообразии сил и интересов, действующих в инновационной среде, существуют несколько ключевых факторов (слоев, сфер модели), без наличия и активной деятельности которых невозможна инновационная деятельность. Позиция 1 - центр модели, зерно инновации, первая ключевая позиция инновационной деятельности.

Ближайшей средой, окружающей атом инновации, является сообщество ученых, изобретателей и инноваторов (позиция 2). Активность этого сообщества - наиболее важный фактор для формирования атома, стимулятор появления у инноватора намерений к действию. К тому же это сообщество является источником экспертов для будущего проекта и помощников для инноватора на начальных этапах.

Следующий уровень инновационной среды - инновационная инфраструктура (позиция 3), представляющая собой совокупность организаций и людей, помогающих инноваторам осуществлять инновационную деятельность. Необходимость этого слоя обусловлена большим объемом разнообразных задач, которые приходится решать инноватору, подчас не имеющему достаточной квалификации.

Позиция 4 - сообщество инвесторов, работающих в интересах инновационной экономики. Лишь небольшую часть инновационного цикла жизни инноватор может преодолеть за собственные средства, поэтому обязательным условием инновационной модели развития является сообщество инвесторов, заинтересованных в инновационных продуктах. Это вторая ключевая позиция для реализации инновационной деятельности.

Позиция 5 включает сообщество, которое в традиционной модели производства занималось научными исследованиями, опытно-конструкторскими работами и подготовкой кадров. В данном контексте эта сфера рассматривается не как источник инновационных идей, а как проектная деятельность, предваряющая производство. Любая идея должна быть представлена в виде проекта, то есть в виде конструкторской, технологической, рабочей документации, расчетов - всего того, что необходимо для производства продукции. Подготовкой проекта должны заниматься профессиональные конструкторы, технологи, инженеры, экономисты. Сам инноватор может быть очень хорошим специалистом в какой-либо области, но быть высококлассным специалистом во всех областях невозможно. Поэтому важнейшим фактором успеха инновации является готовность научно-проектной среды подхватить чужую идею для конструкторско-технологической подготовки производства.

Кстати, острейшей проблемой инновационной деятельности сегодня как раз и является непонимание инвесторами того, что инвестирование требуется не только в производство продукции, но и в ее подготовку к производству.

Следующая сфера - промышленные предприятия и бизнес-структуры, готовые наладить производство инновационной продукции и реализацию инновационных видов услуг на основе проработанных проектов (позиция 6). Без инновационных проектов у предприятий нет шансов на выпуск конкурентоспособной продукции и на выживание в мировой инновационной экономике. Поэтому этот слой модели, помимо важности для инновационной деятельности, должен быть и наиболее активным для инноваций, так как взаимные интересы инноватора и предприятия достаточно сильны. Это третья ключевая позиция для успешной реализации инновационной деятельности.

Позиция 7 относится к сфере конечного потребления инновационных продуктов. В инновационной модели факторы отношения потребителя к инновации не являются главными для инноватора. В этом, собственно, суть инновационной экономики. Идея рождается не у покупателя, а у изобретателя. Однако для предприятий (позиция 7) и инвесторов (позиция 4) возможность продажи инновационного продукта - главный фактор для принятия решений. Поэтому намерения производителей и инвесторов всегда будут содержать жесткие требования к учету этой возможности. Инноватору, не имеющему ни возможностей, ни инструмента работы с мнением рынка потенциальных покупателей, довольно сложно убедить инвестора и предприятия в успешности будущих продаж. Проблема решается информационным взаимодействием всех слоев модели инновационной среды. Если заинтересованность в инновациях в инновационной среде, особенно в ключевых сферах (позиции 4 и 7), достаточно высокая, то изучение и особенно формирование спроса на будущую инновационную продукцию становится делом информационного взаимодействия всех заинтересованных сторон. В этом проявляется благоприятность ситуации в инновационной среде.

Ключевая роль в создании такой благоприятной ситуации принадлежит государственной политике в области инноваций (позиция 8). Намерения государства, заинтересованного в конкурентоспособной экономике, должны выражаться в создании нормативно-правовой базы, инновационной инфраструктуры и целевых программ по финансированию инновационной инфраструктуры и в собственно поддержке инновационной деятельности. Разумеется, финансирование должно осуществляться на начальных этапах структурных преобразований и при запуске новых правил игры.

Последним слоем модели (но не последним по значимости) является состояние мировой экономической системы и мировых достижений в области науки и техники (позиция 9). Учет этого состояния необ- 
ходимо проводить при сформулированных целях и задачах в области инноваций в нашей стране. Важно определиться, будет ли Россия ориентироваться на собственные инновации или основным источником станут иностранные достижения и какие комбинации возможны при использовании своих и иностранных инноваций.

Все перечисленные факторы в большей или меньшей степени влияют на намерения инноватора относительно инновационной деятельности: начинать ее или нет.

Позиции 1, 4, 7 являются ключевыми, потому что именно в этих точках принимаются решения о продолжении развития инновации.

Разумеется, рассмотренные факторы инновационной среды не являются исчерпывающими. Практика показывает, что существует множество факторов, влияние которых трудно учесть заранее, однако нередко именно они имеют определяющее значение при принятии решений о поддержке или остановке инновационного проекта.

Обозначим всю совокупность этих факторов как Х-факторы. К ним могут относиться политические, демографические, социальные и другие факторы. Например, открытия и изобретения в сфере нетрадиционных источников энергии могут встречать сильное сопротивление со стороны сырьевых компаний. Неудачный инновационный проект может неожиданно получить сильную поддержку по причине родственных связей. Существует множество других ситуаций, которые встречаются очень часто, но не могут учитываться как решающее правило при построении модели.

\section{Инновационный проект}

Понятие «инновационный проект» гораздо шире общепринятого понятия «проект». К основным определениям проекта можно отнести следующие.

Проект - замысел, идея, образ, воплощенные в форме описания, обоснования, расчетов, чертежей, раскрывающих сущность замысла и возможность его практической реализации.

Проект - это работы, планы, мероприятия и другие задачи, направленные на создание нового продукта (устройства, работы, услуги).

Несмотря на довольно широкое толкование понятия «проект», для инновационного проекта этого недостаточно.

Во-первых, традиционный проект подразумевает предварительное планирование работ, что в инновационной деятельности крайне затруднительно [1, 2], по крайней мере, традиционными методами.

Во-вторых, традиционный проект подразумевает только описание в какой-либо форме - само изделие и его дальнейшую судьбу редко относят к понятию проекта. Особенность инновации как продукта заключается в том, что свойство инновационности проявляется на этапах и производства, и конечного потребления. Когда инновационность исчезает, инновация становится обычным товаром и все преимущества инновации пропадают. Потребителю в общем-то безразлично, инновационный товар или нет - он приобретает выгоду (полезность) с лучшими характеристиками, и наклейка «инновационный» носит для него чисто рекламный характер. А для инноватора, инвестора и предприятия инновационность является основой конкурентоспособности и эффективности, поэтому для них продление инновационных свойств на весь цикл жизни является важной задачей.

Таким образом, можно утверждать, что инновационный проект - более широкое и более сложное понятие.

Разделение понятий целесообразно еще и потому, что использование устоявшихся терминов и определений очень часто приводит к проблемам взаимного непонимания заинтересованных сторон, когда новая ситуация или проблема описываются с помощью старых форм. Именно это постоянно и происходит сегодня в инновационной деятельности.

Инновационный проект характеризуется не планированием работ, а фиксацией состояния и оценкой объекта. Поэтому важно знать механизм формирования инновации в каждый момент времени инновационного цикла жизни. Фиксация промежуточных состояний инновации по сути и есть инновационный проект.

Необходимость введения понятия «атом инновации» в инновационном проекте обусловлена тем, что на этапах инновационного цикла жизни происходит трансформация как самой идеи, так и отношения к ней заинтересованных сторон. На предпосевном этапе, когда инноватор только начинает формализовать идею, он работает самостоятельно, то есть сфера его интересов лежит исключительно в описании идеи. В гораздо меньшей степени он занимается вопросами оценки своего потенциала и состояния внешней среды. В таком состоянии инновационная идея мало кого может заинтересовать в плане финансирования и поддержки, поэтому целесообразно представить состояние инновации на этом этапе как атом. Атомы должны собраться в «молекулу» инновации. Молекула представляет собой описание инновации с учетом возможностей и намерений заинтересованных сторон внешней (инновационной) среды. Первая молекула формируется уже на посевном этапе, когда появляется первый инвестор, проявивший интерес к иннова- 
ции. Требования этого инвестора (его атома) и являются условием формирования первой (простейшей) молекулы инновации. В этих требованиях могут содержаться и пожелания по описанию состояния внешней среды.

А что же такое инновационный проект?

Если инновационная идея - сущность нематериальная и закрытая для внешнего мира, то проект - материализация идеи через ее описание. Поэтому можно сказать, что проект появляется с началом описания идеи. И проект, в свою очередь, состоит из описания атомов и молекул инновации. То есть под инновационным проектом будем понимать все составляющие описания инновации, соответствующие данному проектному этапу.

Необходимость в такой не совсем традиционной модели описания инновации станет понятна позже, когда будут рассматриваться методы оценки инновационного проекта через математический аппарат фракталов.

\section{Описание атома инновации}

Общее понятие об атоме инновации было сформулировано выше.

Важно еще раз подчеркнуть, что тройка УЖВ - это потенциальные свойства информационночеловеческих элементарных частиц. Они обладают неким дуализмом. С одной стороны, это потенциальные возможности, намерения, некие энергетические состояния без материального воплощения, с другой, в каждый момент времени они могут быть обнаружены, зафиксированы и оценены (то есть в некотором смысле материализованы).

Прошлые умения могут быть выражены через общий уровень образования, профессиональные успехи. Желание может быть оценено через анкетирование, косвенную оценку предыдущей творческой активности, а возможности - через описание окружающей инновационной среды и статистику ее использования.

Потенциальность свойств этих элементарных частиц проявляется в том, что при одних условиях начинается некоторое движение (формирование инновационных молекул), а при других нет. То есть, с одной стороны, проявляется объективность ситуации (некоторые не зависящие от человека факторы), а с другой, проявляется ее субъективность, то есть то, как сам человек оценивает текущую ситуацию и состояние.

Другими словами, движение начнется только тогда, когда внешние и внутренние условия атома совпадут по направлению и оценке.

Рассмотрим пример. Инженер на заводе, безусловно, обладает потенциалом к изменениям. Он может быть достаточно образованным и иметь опыт работы, видит и знает, что можно изменить к лучшему как в организации работ, так и в выпускаемой продукции. В глубине души он может хотеть рассказать комулибо о возникших у него идеях. Оценивая возможности раскрытия своей идеи миру, ему приходится выбирать между следующими альтернативами.

Возможно, инициативу оценят и поддержат. Тогда это повлечет за собой карьерный рост, рост доходов, признание его ценности.

Инициатива может быть воспринята как попытка подсидеть начальника, указать на некомпетентность некоторых сослуживцев, как отвлечение от основных обязанностей в ущерб качеству основной работы. Тогда неизбежны наказание, потеря дохода, снижение комфортности условий работы.

Возможен и третий вариант - уход в самостоятельное плавание, самостоятельная инновационная деятельность.

Это внутренние оценки тройки УЖВ относительно возникшей идеи.

Внешние условия, создаваемые объектами инновационной (или неинновационной) среды (например, правила работы на данном заводе), могут быть благоприятными или неблагоприятными для публичного выражения своих идей инженером. Даже если на заводе продекларирована поддержка творческой активности (что часто бывает с высоких трибун), реальная обстановка может оцениваться работником (инженером) как неблагоприятная для открытого высказывания идей о необходимых изменениях. Исходя из совокупности внешних и внутренних условий, инженер принимает решение о том, выпускать идею «на волю» или нет.

На основании примера можно сделать следующие выводы.

Во-первых, должен быть достаточно высокий потенциал УЖВ, чтобы идея пришла в голову и была готова к озвучанию.

Во-вторых, должны быть соответствующие внешние условия, чтобы идею можно было высказать.

В-третьих, оценка внутреннего потенциала и внешних условий производится субъективно человеком, то есть с разной степенью адекватности реальному положению дел. Эта оценка может быть и оптимистичной, и пессимистичной. 
В-четвертых, запуск инновационной модели развития возможен только при истинном (а не формальном) совпадении интересов субъекта (человека) и внешней среды (предприятия, государства).

Повсеместно на разных уровнях можно услышать утверждение, что инновационных проектов нет, поддерживать нечего, никто ничего не предлагает, никто ничего не хочет. Попытка разобраться в истинности таких утверждений - одна из целей данной статьи.

Еще раз остановимся на дуализме атома инноваций.

Изначально тройка УЖВ представляет собой некое информационное поле, присущее только разуму человека. Несмотря на некоторую объективность УЖВ, важно, как человек оценивает для себя состояние этой тройки. Например, умение может выражаться в многолетнем опыте, твердых навыках, приобретенном мастерстве, но человек может считать, что для обнародования его идей и предложений этого недостаточно.

Поэтому тройка УЖВ изначально является лишь потенциалом информационно-человеческого поля, некой нематериальной энергетической субстанцией.

На рисунке 4 показан процесс перехода потенциала инновационной идеи в атом инновации. В позиции 1 тройка УЖВ представляется лишь потенциалом человека к генерации идеи. Этот потенциал должен достичь некоторого порогового значения, чтобы у человека сформировались намерения к действию - формализации появившейся идеи. Намерения (позиция 2) являются условием преобразования поля в материальную частицу - атом (позиция 3). Материальной она является в том смысле, что появляется первое формальное описание идеи, которое может посмотреть (и понять) другой человек.

Именно в атоме тройка УЖВ и идея приобретают форму, которая может быть оценена другим человеком.



Безусловно, каждый человек имеет свой потенциал и свои идеи относительно изменения окружающего мира. Это проявляется в быту, в учебе, в спорте, на работе, то есть повсеместно. Каждый человек, чаще или реже, что-то хочет изменить в своем окружении, но не каждый проявляет намерения поделиться своими мыслями с окружающими, а тем более изложить их в какой-либо форме.

Таким образом, атомы могут формироваться только при определенных условиях: достаточно высоком внутреннем потенциале и созревших намерениях к каким-либо действиям. Они появляются тогда, когда в человеке просыпается творец, созидатель, когда желание действовать становится сильнее чувств опасности и риска, связанных с продвижением идеи.

Можно провести аналогию с базовыми понятиями маркетинга - нуждой и потребностью [5]. Нужда формируется под действием объективных факторов (голод, холод), а потребность под действием факторов нужды и субъективных факторов, например, таких как предлагаемые товары и способы удовлетворения нужды. Человек, оценивая нужду как некую необходимость и принимая во внимание внешние предложения и условия, формирует в своем сознании конкретную потребность. Эта потребность и является началом действий потребителя по поиску и оценке подходящих товаров и услуг.

В инновационной модели такой нуждой является потенциал информационно-человеческого поля, а потребностью - намерение к действиям по продвижению идеи. Это намерение основывается, с одной стороны, на величине внутреннего потенциала, а с другой - на оценке состояния внешней среды (благоприятной или нет).

Можно привести аналогию и из области электротехники. Напряжение электрического поля создает лишь потенциал для электрического тока. Но достаточным условием возникновения тока является величина сопротивления. При высоком сопротивлении тока нет, при низком ток есть. В данном случае намерение можно охарактеризовать как оценку человеком отношения величины собственного потенциала к сопротивлению внешней среды. В одном случае намерение действовать появляется, в другом нет.

Разница понятий «потребность» и «намерения» в маркетинговом и инновационном подходах заключается в том, что потребитель хочет взять готовое, а инноватор дать новое. В первом случае потребитель заранее может точно сказать, что он хочет взять, во втором случае потребитель не знает заранее, что он будет брать. В работе [2] проведена аналогия между деятельностью маляров и художника. Малярам по- 
требитель может точно сказать, за что он готов заплатить. В случае с художником потребитель заранее не знает, купит ли он картину, пока не увидит ее и не оценит.

Таким образом, намерение является важнейшим условием начала инновационной деятельности и процесса кристаллизации идеи в инновацию.

\section{Функции и свойства идеи}

Как уже было сказано, с системной точки зрения инновационные атомы состоят из инновационной идеи (ядро атома) и информационно-человеческого поля атома (тройка УЖВ).

Инновационная идея в общем случае - это набор функций и свойств, функция - способность (возможность) решать какую-то задачу, свойства - характеристики, позволяющие реализовать эту возможность.

Функция - это новая полезность, реализуемая новым способом, устройством, веществом. Суть функции всегда может быть выражена кратко. А реализация этой функции требует обстоятельного описания через множество свойств и характеристик.

Уместно вспомнить правила оформления изобретений. Формула изобретения требует изложения его сути одним предложением (причем по шаблону). Формула изобретения - это и есть краткое описание функции (как новой полезности). А описание изобретения - это описание свойств элементов, из которых будет формироваться собственно изобретение.

Функций в общем случае может быть и несколько, но всегда должна быть главная. Функции подразделяются на внутренние и внешние. Внешняя функция направлена на создание полезности для внешнего мира, внутренняя - на реализацию устройства инновационной идеи, то есть каким образом будет реализована эта идея. Весьма полезным в этом смысле является методология функционально-стоимостного анализа (ФСА) [6].

Функции и свойства идеи - это умозрительные, невещественные субстанции. Материализация функций и свойств - описание их в какой-либо форме. Материальность описания заключается в том, что, несмотря на отсутствие самой инновации (как материальной реализации идеи), идею и будущую инновацию уже можно понять, оценить и обсчитать, а затем лишь отобразить описание в какую-то материальную форму (что является делом техники).

Поэтому, когда речь идет об атоме инновации, подразумевается уже некая материальность идеи в смысле ее понимания заинтересованными сторонами.

Необходимо сказать и об отличии научной идеи от инновационной. По сути они практически не отличаются друг от друга ни по способу появления, ни по тройке УЖВ. Разница проявляется в степени учета состояния внешней среды.

Научная идея, как правило, самодостаточна. Публикация идеи (в разной степени проработанности) и есть конечная цель научной деятельности. Дальнейшая судьба идеи, как правило, мало волнует ученого. Инновационная идея, наоборот, после первой формализации (не всегда публикации) находится в самом начале цикла своей жизни. Это нулевой шаг в создании инновации, а таких шагов на всем цикле жизни надо сделать еще очень много.

Идея становится инновацией только тогда, когда в ее описание попадают не только функции и свойства идеи, но и внешняя среда, в которой будет разрабатываться, производиться и использоваться инновация. Одно из основных заблуждений прикладной науки в том, что она занимается созданием инноваций. Наука генерирует новые идеи (знания), которые автоматически никогда не станут инновациями.

Это же относится к изобретениям и к изобретателям. После получения патента на изобретение у них есть выбор: остаться изобретателем или стать инноватором.

\section{Упакованность атомов}

Когда говорят об атоме инновации, подразумевают, что он представляется для заинтересованной стороны через свое описание.

Описание является полным (упакованным), если заинтересованная сторона полностью понимает его (с точки зрения принятия каких-то решений).

Описания атомов инноватора отличаются от описаний атомов других заинтересованных сторон степенью полноты.

Только атом инноватора имеет законченную материальную форму, по которой можно понять и оценить инновацию, то есть саму идею, потенциал инноватора и факторы инновационной среды, описанные в какой-либо форме (см. таблицу). Форма и степень детализации определяются этапом цикла.

Атомы остальных заинтересованных сторон представлены своим потенциалом и намерениями. 
Описание атома инновации инноватора

\begin{tabular}{|l|l|l|}
\hline \multicolumn{1}{|c|}{ Идея } & \multicolumn{1}{|c|}{ Тройка УЖВ } & Факторы инновационной среды \\
\hline Назначение & Умение - фиксация профес- & Описание факторов внешней \\
Функции: внешние, внутренние & сиональных достижений & (инновационной) среды, \\
Свойства (характеристики): & инноватора & благоприятствующих \\
внутренние, внешние & Желание - фиксация & и препятствующих \\
Структурная модель: состав & мотивов инноватора & инновационной деятельности \\
и взаимосвязь элементов & Возможности - фиксация & и созданию самой инновации \\
& условий, позволяющих & \\
& начать и продолжить работу & \\
& с инновацией & \\
\hline
\end{tabular}

Чем отличается упакованный атом от неупакованного?

Описание неупакованного атома заканчивается намерениями. То есть инвестор декларирует набор свойств проекта (инновации, идеи) в виде пожеланий (намерений) как условий поддержки. Грубо говоря: «Я готов поддержать хороший проект, хорошего инноватора при условии, что найдется хорошее предприятие, реализующее инновацию». В данном контексте «хороший» - это намерение, которое никак не может быть определено без рассмотрения конкретной инновации.

А атом инноватора должен содержать не только набор декларированных свойств (характеристик, параметров), но и значения этих параметров (характеристик), причем желательно в числовой (количественной) оценке.

Здесь уместна аналогия с объектно-ориентированным программированием [7].

Если атом представить в виде класса объектов, то атом инноватора будет объектом класса с определенными и заданными свойствами, а атомы других заинтересованных сторон остаются классами с декларированными возможными свойствами.

Эта аналогия может быть весьма наглядной, так как здесь наблюдается много схожих механизмов в функциональных и параметрических описаниях информационной модели инновации. Сложность заключается в обязательном присутствии в этой модели принимающих решения лиц с не всегда рациональной и предсказуемой логикой.

Есть и другая сторона этой проблемы. Что для инвестора важнее в атоме инноватора: идея, личность инноватора или факторы внешней среды? Ответ не так очевиден, как может показаться на первый взгляд. Казалось бы, хороший проект имеет безусловный приоритет. Но чего стоит такой проект, если некому вести его по этапам? Ведь он постоянно детализируется во времени (процесс проектирования). Вероятность успешного завершения проекта без его инициатора (его знаний и предпринимательской активности) резко снижается. С другой стороны, хороший инноватор может инициировать и довести до реализации множество проектов, даже если какой-то конкретный проект будет не самым удачным. Решающим фактором в принятии решений о поддержке конкретного проекта может быть и готовность внешней (инновационной) среды принять определенный класс инноваций.

Все это говорит о том, что ни инвестор, ни предприятие заранее не могут сформулировать четкие требования (количественную оценку параметров) к инновационным проектам, которые будут обязательно поддержаны, как и в случае с художником невозможно сформулировать критерии (и параметры) будущего шедевра.

Поэтому атомы других заинтересованных сторон не могут быть полностью упакованными по определению.

Атомы других заинтересованных сторон также можно назвать атомами инновации, хотя они не имеют прямого отношения к инновационной идее. Но, как уже отмечалось, инновационная идея отличается от научной тем, что она обязательно учитывает состояние внешней среды. Без этого атомы не соберутся в молекулу, а затем в полноценный проект. Влияние атомов среды на атом инноватора обязательно. Именно информационное взаимодействие атомов создает предпосылки к их последующему объединению.

Далее по тексту вместе с термином «атом инновации» будет использоваться также сочетание «атом инноватора» либо «атом какой-либо заинтересованной стороны» (например атом инвестора).

\section{Функции и свойства атомов}

В контексте данной статьи атомы - это наименьшие частицы какой-либо деятельности. Например, в традиционной модели экономики таким атомом является операция (раздел экономической науки «операционный менеджмент»). Это элементарные функции деятельности, из которых складывается собственно 
вся деятельность. Например, производственные функции, коммерческие функции, инвестиционные функции.

Инновационный атом появляется в том месте, где предполагаются изменения деятельности. В этом смысле в любой деятельности могут появиться свои инновационные атомы как ответ на потребность в изменениях в производственной, инвестиционной и любой другой деятельности. Инновационные атомы могут присутствовать во всех видах деятельности, существуя параллельно с традиционными. При этом функции инновационных атомов в одних случаях будут совпадать с видом деятельности (виды деятельности, связанные с проектированием новой продукции), в других не будут совпадать с основным видом деятельности (например в производстве).

В условиях инновационной экономики отсутствие инновационных атомов в любом виде деятельности приводит к потере конкурентоспособности и эффективности. Задачи конкурентоспособности и эффективности требуют создания механизма, формирующего из инновационных атомов инновационные молекулы, из молекул - полноценные инновационные проекты, а из проектов - инновационную продукцию и услуги.

Но сама инновационная деятельность может проходить в нормальном режиме только тогда, когда обеспечивающие виды деятельности работают в нормальном неинновационном режиме, то есть инвесторы инвестируют, производственники производят, продавцы продают. Поэтому, когда речь идет об атоме инвестора, значит, в этом атоме реализуется основная, а не инновационная функция.

Чтобы инновационная деятельность была начата, принципиально наличие трех сторон: инноватор (инициатор идеи, разработчик инновации, инновационный предприниматель, ученый, изобретатель), инвестор, потребитель инновации (предприятие, следующий инвестор) [2].

Остальные стороны внешней (инновационной) среды могут существенно влиять на состав проекта на протяжении всего инновационного цикла жизни, но именно для начала проекта принципиальными являются вышеперечисленные стороны. Эти три атома (первых трех заинтересованных сторон) и составляют первую простейшую молекулу инновации (начало инновационного проекта).

На рисунке 5 показаны обязательные заинтересованные в появлении инновационного проекта стороны. В проекте начального этапа (посевного) как раз и отражаются интересы этих трех сторон, то, без чего невозможны начало и движение инновации по инновационному циклу жизни. В качестве инвестора (как функции) может выступать не только специализированная организация - эту функцию могут выполнять и отдельное предприятие (кластер предприятий), и частные лица, и любая организация, выделяющая средства на внутренние инновационные изменения.

Чтобы сформировалась молекула инновации (начальный инновационный проект), необходимо пройти несколько этапов построения информационного инновационного пространства инновации. Инновационный проект - это сложный информационный продукт, состоящий из инновационных атомов и молекул. Силы притяжения атомов создают потенциальную возможность построения инновационных молекул, а те, в свою очередь, с помощью некоего «клея» соединяются с другими молекулами, складываются в полноценный инновационный проект.

Инновационная деятельность протекает в условиях постоянного поэтапного формирования информационного инновационного пространства.

На первом этапе формируется описание инновационного пространства на атомном уровне. В общем случае началом инновационного проекта можно считать первое описание, включающее предлагаемую идею, представление разработчика инновации, интересы заинтересованных сторон (инвестора и потребителя инновации).

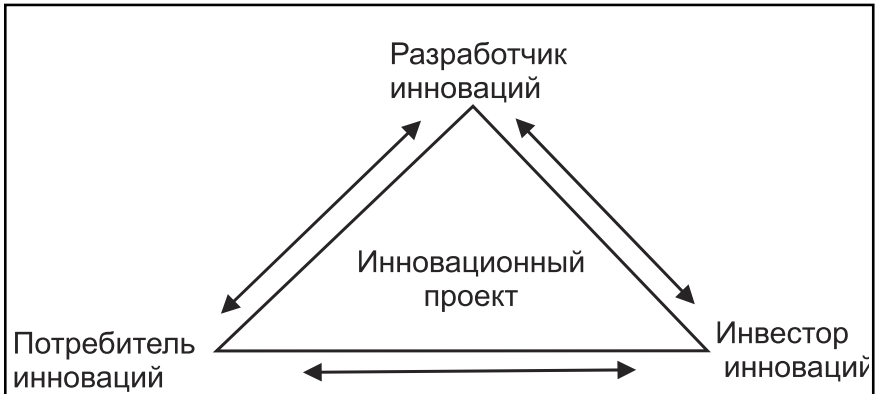

Рис. 5. Начало инновационного проекта
Это описание можно представить в виде треугольника интересов (рис. 5), в каждой вершине которого находится атом, относящийся к определенному виду деятельности.

Интересы сторон представляются своими атомами, взаимное притяжение которых создает простейшую молекулу инновации - начальный инновационный проект: атом инноватора (Ан), атом инвестора (Аи), атом потребителя (Ап) инновации (рис. 6).

На рисунке 7 представлен атом инноватора (Ан) с его тройкой: умение инноватора $\left(\mathrm{У}_{\mathrm{H}}\right)$, желание инноватора (Жн), возможность инноватора $(\mathrm{BH})$.

Главной функцией является Ан - продвижение идеи по инновационному циклу жизни, остальные функции (доход, признание, удовлетворение) второстепенные. 
Ун складывается из уровня профессиональных знаний и навыков (образование, опыт, кругозор), аналитических способностей (способность понять и сформулировать проблему, решение), способности обоснования и представления идеи (выбор выразительных средств, способность произвести расчеты, построить модель и т.п.).

Жн складывается из потребности в признании и в увеличении дохода, из отношения к риску и неопределенности.

Вн складываются из оценки благоприятности внешней среды (наличие доступных элементов инновационной инфраструктуры заинтересованных сторон, понятные правила иг-

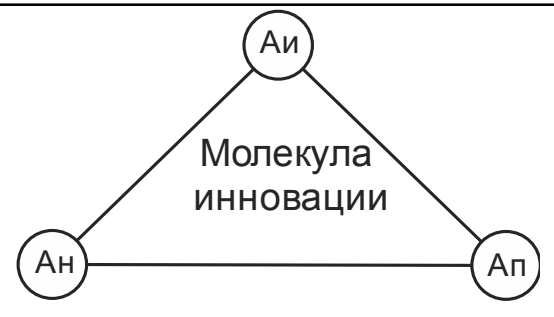

Рис. 6. Простейшая молекула инноващии ры), предпринимательских способностей, понимания своевременности творческой активности (не опережая требования внешней среды и не отставая от них).

На рисунке 8 показан атом инвестора (Аи) с его тройкой: умение инвестора (Уи), желание инвестора (Жи), возможности инвестора (Ви).

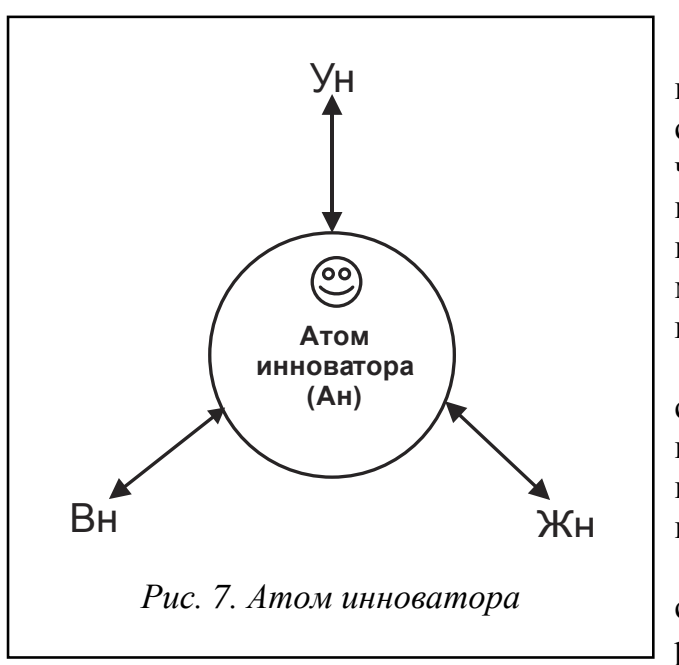

Главная функция Аи - возврат инвестиций с прибылью.

Уи складывается из уровня знаний и навыков (образование, опыт, кругозор) в сфере инвестирования, аналитических способностей в понимании и содержательной оценке чужих идей, способности к стоимостной оценке полученных и будущих результатов интеллектуальной деятельности инноватора и интеллектуальной собственности, способности модификации идеи инноватора в сторону построения выгодной бизнес-модели.

Жи складывается из потребности в увеличении дохода от сделанных инвестиций, осознания полезности инвестиционной деятельности (инвестирование от лица государства, представление интересов более крупного инвестора), из отношения к риску и неопределенности.

Ви складывается из оценки благоприятности внешней среды (наличие инновационной инфраструктуры и заинтересованных сторон, понятные правила игры, благоприятная политика государства), наличия подходящей нормативноправовой базы и достаточных денежных средств или других форм и методов инвестирования в инновационные проекты.

Разумеется, в рамках одной статьи список факторов, влияющих на потенциальные свойства УЖВ, не может быть полным, но для построения модели он вполне достаточный.

Инвестирование не является совершенно новым видом деятельности в отличие от инвестирования в инновационную деятельность - абсолютно нового, еще не поддержанного методически вида. Дело в том, что традиционные методики инвестирования ориентированы на существующую бизнес-ситуацию. Это описание текущей ситуации, формулирование проблем и предложение по инвестированию, которое улучшит показатели именно в существующей ситуации. Инновационная деятельность подразумевает будущие товары и будущую бизнес-ситуацию. Инвестирование в данном случае не улучшает, а создает новую бизнесситуацию. Поэтому методики традиционного инвестирования в общем случае не подходят к задачам инновационного инвестирования и неизбежно появление инновационных атомов и в инвестиционной деятельности.

На рисунке 9 представлен атом потребителя инновации (Ап) с его тройкой: умение потребителя (Уп), желание потребителя (Жп), возможность потребителя (Вп) инновации.

Главная функция Ап - получение конкурентоспособности.

Под потребителем инновации понимается сторона, которая пытается извлечь выгоду из инновации, частично или полностью приобретая права на нее. Так как инновационный цикл жизни состоит из многих проектных этапов [2], потребителем инновации каждый раз могут быть разные субъекты и объекты. Инновационный проект может передаваться (продаваться) другой инновационной компании для дальнейшей разработки, может предлагаться следующему инвестору или предприятию для реализации про- 


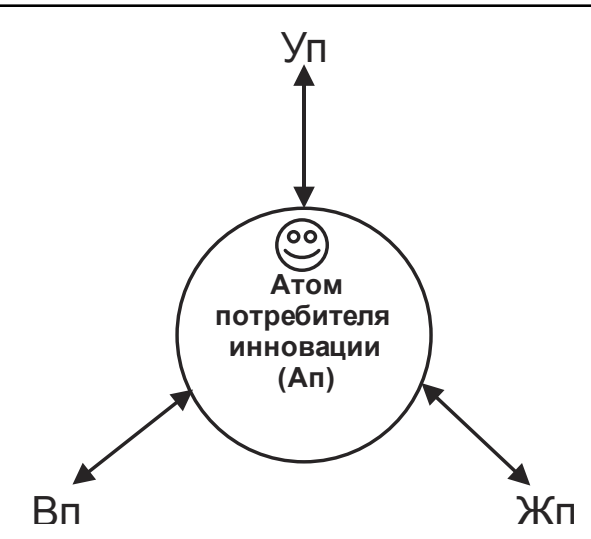

Рис. 9. Атом потребителя инноващии екта в товар (с передачей прав на интеллектуальную собственность). Рассматривать конечного потребителя в качестве потребителя инновации нецелесообразно, так как покупатель приобретает товар с лучшими функциями и свойствами и ему в общем случае безразлично, инновационный товар или нет.

При рассмотрении предприятия как потребителя инновации подразумевается, что решение принимает конкретный человек, представляющий интересы своего предприятия (это же касается и других заинтересованных сторон), и этот человек обладает своей тройкой УЖВ.

Уп складывается из уровня профессиональных знаний в области производства своего предприятия (образование, опыт, кругозор), аналитических способностей (понимание перспектив, знание достижений науки и техники, приоритеты рынка, политика поддержки государства), способности оценивать затраты на технологические изменения при реализации инновационного проекта.

Жп складывается из потребности в конкурентоспособной продукции и в повышении конкурентоспособности и эффективности своего предприятия, из отношения к риску и неопределенности.

Вп складываются из оценки благоприятности внешней среды (возможности создания спроса на инновационную продукцию, политика государства в области инноваций), наличия инвесторов, берущих на себя риски и затраты по поддержке инновационных проектов, возможности приобретения прав на интеллектуальную собственность.

Еще раз подчеркнем, что это внутренние потенциальные свойства инновационных атомов. Наличие потенциала не является гарантией начала инновационного проекта, это лишь необходимое условие его появления.

\section{«Клей» молекулы. Силы притяжения}

Как уже было сказано, атомы - это лишь потенциальные возможности генерации новых идей или изменений. Сами по себе, без соответствующих условий (усилий субъектов и формальных описаний) они не собираются в молекулы. Так как инновационная деятельность - информационный процесс и в нем одновременно участвуют несколько сторон, то он может происходить только при условии постоянной формализации (некоторой формы описания и использования выразительных средств), а также в осознании и убежденности в целесообразности поддержки проекта.

Интересы заинтересованных сторон настолько разные, что при описании атомов в проекте надеяться на их автоматическое совпадение не приходится. Необходимы дополнительные факторы (информационные составляющие), которые приведут к общему знаменателю описания (намерения, требования) различных сторон. Необходим некий «информационный клей», позволяющий стянуть и закрепить различные атомы в молекуле. Отсутствие этого «клея» приводит к тому, что описания атомов инновации в проекте носят фрагментарный характер и не производят нужное впечатление на заинтересованные стороны, то есть не обладают обоснованностью и убедительностью для принятия необходимого решения.

Второй этап построения информационного инновационного пространства - описание инновационной молекулы. Построение инновационной молекулы - это привлечение внимания заинтересованных сторон

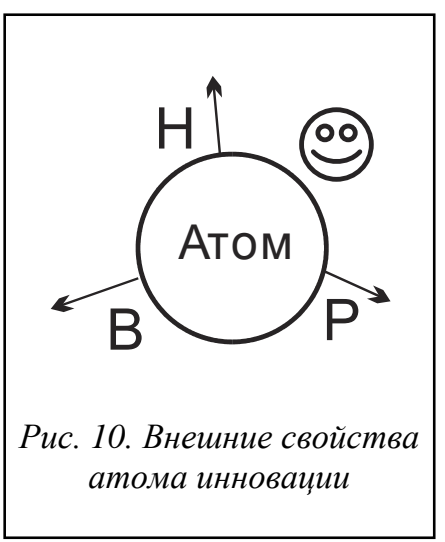
к идее инноватора. Привлечь внимание можно, если представить формальное описание идеи в формате, понятном и интересном для инвестора и потребителя инновации. Это позволит создать первые силы притяжения между атомами (Ан, Аи и Ап).

Для этого у атома инновации (в качестве «клея») должны быть сформулированы следующие внешние свойства: новизна $(\mathrm{H})$, реализуемость $(\mathrm{P})$, выгодность (B).

На рисунке 10 показана тройка внешних свойств (НРВ) атома. Значок (;) указывает на субъективный характер описания внешних свойств, то есть именно так, как представляет себе, например, инноватор, должна выглядеть идея для внешнего мира, чтобы быть привлекательной. Разумеется, это описание формируется и под воздействием требований другой заинтересованной стороны (например инвестора). В этом и есть основа силы притяжения и будущего «клея» для сближения и фиксации атомов. 

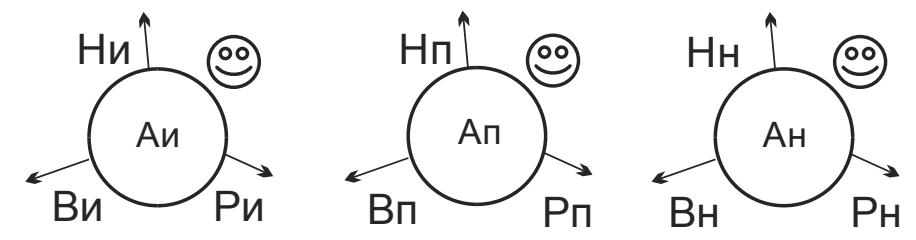

Рис. 11. Внешние свойства атомов инвестора, потребителя инновачий и инноватора

ции, которые предлагаются ему на рассмотрение и оценку.

Нп, Рп, Вп - соответственно внешние свойства атома потребителя инновации: новизна, реализуемость и выгодность. Это то, как представляет себе потребитель инновации (предприятие) внешние (полезные для предприятия) свойства атома инновации.

Нн, Рн, Вн - соответственно внешние свойства атома инноватора: новизна, реализуемость и выгодность. Это то, как представляет себе инноватор внешние (полезные для внешнего мира) свойства атома инновации.

Привлекательность инновационного проекта обусловлена совпадением именно именно этих внешних свойств атомов.

Внешние свойства атомов (НРВ) очень важны на этапе зарождения молекулы инновации - первого описания идеи инноватора с учетом интересов инвестора и потребителя инновации (первое представление об этих интересах). Это по сути бизнес-модель будущего товара, услуги или вида деятельности (первый вариант бизнес-модели).

Описание НРВ атомов всех заинтересованных сторон позволяет запустить информационный процесс поиска и оценки идей. В условиях рыночной инновационной экономики потребность в инновационных идеях очень высока. Через поток инновационных идей и проектов решаются как частные задачи инноваторов, инвесторов и предприятий, так и общие задачи государства по повышению эффективности экономики в целом.

У инвестора и потребителя инновации (предприятия) свойства НРВ носят декларативный характер (что хотелось бы увидеть в инновации). У инноватора свойства НРВ носят описательный характер (описание будущего товара или услуги в виде набора функций и свойств, полезных для конечного потребителя).

Рассмотрим свойства НРВ для каждой заинтересованной стороны.

Свойства НРВ инноватора.

Новизна идеи инноватора (Нн): абсолютная или относительная новизна идеи, возможность правовой защиты идеи (потенциальная возможность), источник идеи (изменения на производстве или новый товар для рынка).

Новизна с точки зрения инвестора (Ни): возможность правовой защиты идеи, степень известности (неизвестности) подхода к решению какой-либо задачи, источник идеи (если идея родилась в недрах предприятия как отражение его задач, определение новизны для внешнего инвестора затруднительно).

Новизна с точки зрения потребителя (Нп) инновации: возможность правовой защиты идеи с точки зрения дальнейшего приобретения прав на интеллектуальную собственность, абсолютная или относительная новизна идеи, внутренняя или внешняя инновация для предприятия.

Реализуемость идеи инноватора (Рн): соответствие идеи текущему уровню развития технологий в мире (может быть подобрана подходящая технология), наличие кадров (потенциальное), способных реализовать идею и довести ее до рынка, возможность появления ресурсов на реализацию идеи.

Реализуемость с точки зрения инвестора (Ри): отсутствие принципиальных ограничений нормативноправового, технологического, политического характера и других ограничений, а также явного потребителя инновации и предпринимательских характеристик у инноватора.

Реализуемость с точки зрения потребителя (Рп) инновации (в частности предприятия): соответствие уровню технологии (близость к существующему уровню технологии предприятия), наличие кадров или возможность их подготовки, достаточность ресурсов для реализации инновации.

Выгодность идеи инноватора (Вн): масштаб предлагаемых изменений (сложность и трудоемкость будущего товара или услуги), возможность развития идеи и получения дохода от реализации для продолжения инновационной деятельности.

Выгодность с точки зрения инвестора (Ви): возможность возврата инвестируемых средств с прибылью, минимальность рисков, возможность формирования «портфельных» инвестиций.

Выгодность с точки зрения потребителя (Вп) инновации: возможность получения конкурентоспособной продукции и повышение конкурентоспособности предприятия, возможность защиты положения на 
рынке (повышение капитализации, защита рыночной доли на рынке, управление спросом, барьеры для конкурентов) и управления финансовыми показателями предприятия (сроки окупаемости, прибыль, масштаб производства).

Перечни свойств не являются исчерпывающими, но их вполне достаточно для построения модели. Дальнейшая детализация возможна в рабочих документах.

Еще раз подчеркнем, что проявление (описание) внешних свойств атомов не является достаточным условием для формирования молекул инновации, а лишь создает силы притяжения, то есть условия для поиска информации заинтересованными сторонами и предварительного анализа потенциальных предложений по новым идеям.

Некоторые внешние свойства (НРВ) у разных сторон практически совпадают, так как они напрямую проецируются на финансовые вопросы (затраты, прибыль). Но некоторые не совпадают и не могут совпадать, так как функции, цели и задачи у сторон в общем случае разные.

Для решения задач, стоящих перед заинтересованными сторонами, требуется постоянная работа по совершенствованию как внутренних, так и внешних свойств атомов, то есть необходимо повышать квалификацию исполнителей, их мотивированность к действию и получению результатов, совершенствовать внешнее представление требований к другим участникам инновационного пространства. Внутренние и внешние свойства атомов влияют друг на друга. Приведение степени их реализации в соответствие некоему эталонному уровню и есть процесс управления инновациями на разных уровнях принятия решений.

Процесс совершенствования внешних свойств при несовпадении интересов сторон (и соответственно формулировок) абсолютно необходим, так как формирование потока инновационных проектов - это и есть источник решения общегосударственных экономических задач. Переход от сырьевой модели экономики к инновационной требует определения своего места в мировой производственной системе. Отсутствие высокоинтеллектуальной составляющей в добавленной стоимости приведет к неизбежной деградации отечественного производства. К сожалению, в настоящее время наблюдается «паралич воли» у всех сторон инновационного процесса. Каждая из них не идет навстречу другим, а ждет указаний сверху, особого финансирования, особого законодательства и еще многого другого. Это является наследием традиционной экономики, когда без указаний сверху ничего нельзя было предпринимать.

Процесс совершенствования внешних свойств заключается в следующем.

Стороны, заинтересованные в понимании целей и задач друг друга, должны находить общий знаменатель при оценке внешних свойств своих атомов. Таким общим знаменателем должны быть свойства инновационной идеи.

Они могут быть измерены с помощью аппарата шкалирования на основе теории полезности. Согласованные между сторонами значения свойств образуют шкалу оценки этих свойств. Отображение значений свойств предлагаемой идеи на эту шкалу позволяет сторонам оценить предлагаемую идею.

Например, выгодность предприятия как получение конкурентоспособной продукции должна быть отображена на шкале оценки полезности инновационной идеи с этой точки зрения. На эту же шкалу отражается выгодность инвестора как возможность возврата инвестиций с прибылью. Совпадение оценок на шкале полезности идеи позволяет утверждать, что внешние свойства атомов предприятия и инвестора по этому критерию имеют близкие ожидаемые значения.

Несовпадение ожидаемых значений указывает на необходимость корректировки выставляемых требований или на то, что предлагаемая идея не подходит какой-либо из сторон.

Разумеется, процедура построения шкал оценки и сама оценка не являются тривиальными, но формализация этой процедуры позволит начать процесс понимания и сближения интересов трех сторон в деле формирования инновационного проекта, удовлетворяющего всем требованиям. В результате количественного измерения информации по свойствам, имеющим качественный характер, появится возможность измерения общей полезности инновационной идеи с точки зрения начала инновационного цикла жизни и вероятности успешного завершения проекта.

Первый и второй этапы формирования инновационного пространства заканчиваются наличием атомов с внутренними и внешними свойствами, создающими ситуацию готовности к движению, но для начала движения нужны еще дополнительные факторы упаковки проекта.

\section{Молекула инновации}

Третьим этапом формирования инновационного пространства для инновации является формирование упакованной молекулы инновации (инновационного проекта, соответствующего проектному этапу). Упакованность подразумевает вписывание инновации в требования ближнего окружения внешней среды. Эти требования могут быть весьма разнообразными, но для модели их можно свести к трем крупным факторам. 
На рисунке 12 представлена модель укрупненных факторов ближнего окружения инновации - источников потребностей и объектов для изменений.

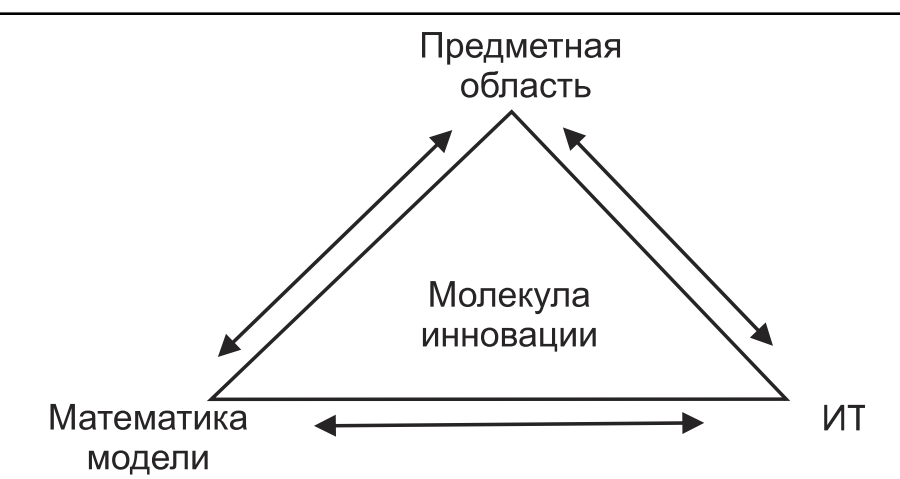

Рис. 12. Требования внешней среды для молекуль инноващуии
Под предметной областью в данном случае понимается любой вид деятельности, в которой предполагаются изменения или внедрение инноваций. Сегодня трудно представить себе деятельность, для эффективности которой не использовался бы математический или методический аппарат. Это вторая вершина треугольника. То есть для успешности изменений в предметной области необходимо менять и организационно-методические модели ведения вида деятельности. Организация современного производства (и любого другого вида деятельности) сегодня подразумевает использование информационных технологий (ИТ). Это своеобразный упаковщик математического и методического обеспечения любого вида деятельности (в разной степени). Как правило, полноценный инновационный проект должен включать изменения не в одной, а во всех трех вершинах треугольника, так как изменения лишь в одной вершине будут встречать сильное сопротивление в остальных вершинах, поддерживающих старую модель деятельности. Именно этой особенностью обусловлено возрастающее значение ИТ в современном производстве (в виде деятельности) в условиях растущих требований к конкурентоспособности. Если ИТ почему-то не используются в предложениях по инновации, то они обязательно должны присутствовать в процессе распространения информации об инновации, в формировании спроса на нее и в других информационных процессах, без которых невозможен инновационный цикл жизни.

Разумеется, сказанное в большей мере относится к инноватору как инициатору идеи, но это имеет влияние и на оценку идеи другими заинтересованными сторонами.

Таким образом, мы получаем тройку свойств ближнего окружения инновации: предмет (П), математика (М), ИТ. Эта тройка характеризует состояние производственно-экономической среды, в которой должна жить инновация.

Определим место этой тройки в инновационном пространстве инновации (рис. 13).



Рис. 13. Место молекулы инновации в тройке П, М, ИТ

Необходимость вписывания молекулы инновации в тройку П, М, ИТ обусловлена тем, что на первом и втором этапах формирования инновационного пространства рассматривались в основном потенциальные свойства предлагаемой идеи (инновации). На третьем этапе необходимо сформулировать влияние предлагаемой идеи на существующую производственно-экономическую среду, то есть вопросы, связан- 
ные с учетом состояния существующей среды и тех изменений, которые повлечет за собой внедрение предлагаемой инновации. Эти вопросы должны учитываться всеми сторонами при рассмотрении инновации. Вполне вероятно, что среда (тройка П, М, ИТ) потребует и других изменений, что повлечет за собой формирование «портфеля инновационных молекул», которые также необходимо будет увязать в данной тройке. Например, инновационная молекула потребует смены технологии производства (П), что в свою очередь потребует смены оборудования, переподготовки персонала, предварительной работы с рынком, смены организационной модели (М), изменения программного обеспечения (ИТ) и т.п. Все это могут быть новые инновационные идеи - «портфельные инновации». В результате из одной инновационной молекулы может вырасти множество инновационных проектов (вариантов). Этим заканчивается третий этап формирования инновационного пространства предлагаемой инновации.

На четвертом этапе непосредственно формируется упакованный инновационный проект (формальное описание). Цель описания инновационного проекта на этом этапе - получить полное представление не только о самой идее, но и обо всем, что связано с инновационной деятельностью и прохождением инновационного цикла жизни. Следует отметить, что степень детализации описания будет меняться в зависимости от проектного этапа инновационного цикла жизни: предпосевной, посевной, стартап, венчурный.

На начальных проектных этапах описательная информация может носить декларативный и экспертный характер, не противоречащий здравому смыслу и опыту экспертов, представляющих интересы своих сторон. На последующих этапах та же информация будет требовать все большего подтверждения фактическими данными, так как величина затрат и цена вопроса будут возрастать, а риски и неопределенности должны снижаться.

Молекула может иметь следующие функции: способность к защите, потенциально высокая добавленная стоимость, потенциально высокий спрос.

Для полноты картины по инновационному проекту необходимо учесть еще три фактора: наличие инновационной инфраструктуры (Инф), предпринимательские способности инноватора (Пре), благоприятная нормативно-правовая база (Нор).

Описание этой тройки факторов (Инф, Пре, Нор) является достаточным для того, чтобы считать описание инновационного проекта полным для принятия решений.



Рис. 14. Заключительная тройка факторов инновационного проекта
На рисунке 14 представлены заключительные факторы, достаточные для описания инновационного проекта соответствующего проектного этапа.

Предпринимательские качества и навыки у инноватора являются одними из важнейших факторов успешного начала и реализации инновационного проекта. К сожалению, этот фактор до сих пор является декларативным в среде поддержки инновационной деятельности. Как правило, инициаторами инноваций являются ученые, преподаватели, инженеры, изобретатели, не имеющие никакого опыта предпринимательской деятельности. Существующая система поддержки пока никак не способствует подготовке этой стороны инновационной деятельности.

Компенсировать предпринимательскую неграмотность могла бы инновационная инфраструктура совокупность организаций и специалистов, призванных помогать прохождению инноваций по инновационному циклу жизни. Дело в том, что при описании проекта, особенно в середине и в конце инновационного цикла жизни, требуется большой объем специальных знаний в области экономики, маркетинга, менеджмента, ИТ и многое другое. Разумеется, один человек не может владеть всеми этими вопросами на должном уровне, поэтому в организациях инфраструктуры должны быть специалисты, помогающие инноватору решать вопросы с подготовкой и оформлением проектов. Есть еще и более острая проблема отсутствие уже у этих специалистов необходимой подготовки в области инновационной деятельности и инновационных расчетов. Применяя знания из методов традиционной экономики, они приносят больше вреда проекту, чем пользы. Явно не хватает квалификации и знаний специалистам, находящимся на стороне инвестора. Отсутствующие методики оценки инновационных проектов часто заменяют методиками 
оценки инвестиционных проектов, по которым ни один инновационный проект не может быть поддержан в принципе.

Третьим важнейшим фактором является состояние правовой нормативной базы в области инновационной политики как на федеральном, так и на региональном уровне. Именно здесь в полной мере проявляется тотальное непонимание сути инновационной деятельности и поставлены мощные барьеры на самое ее начало. Сегодня только чудом можно выпустить инновационный проект в жизнь. Согласно действующему законодательству, правильная организация инновационной деятельности может повлечь за собой даже уголовное наказание.

\section{Вопросы оценки инновации, инновационного проекта, инновационной деятельности. Фракталы}

Успешность инновационной деятельности определяется, помимо всего прочего, возможностью оценки (измерения) характеристик инновационного проекта. Критерием оценки является соотношение «затраты-ожидаемая полезность». Ожидаемая полезность - это комплексный показатель, отражающий уровень достижения целей заинтересованных сторон. Сюда могут входить и финансовые, и рыночные показатели, показатели повышения эффективности производства и другие.

Традиционные методики оценки проектов здесь не могут применяться непосредственно. Дело в том, что в них используются фактические входные данные. В традиционной экономической модели на основе изученного спроса изначально в маркетинговом плане формулируются финансовые показатели по новому товару. Эти показатели и являются фактическими входными данными для всех этапов традиционного цикла жизни, то есть состав работ, сроки и деньги - это фактические входные данные для различных традиционных методик расчета по этапам цикла жизни.

В инновационной модели фактических данных просто нет (по определению). За неимением других методик нередко используются показатели традиционных методик оценки, но часто не берется во внимание тот факт, что смысл показателей и данных в инновационной модели совсем иной: это прогнозные, предполагаемые данные по будущему проекту и товару, в некотором смысле фантазии инноватора на тему успешности реализации его идеи. Причем сам инноватор вряд ли стал бы фантазировать на экономические темы, но его заставляют делать это другие заинтересованные стороны.

Рассмотрим основные принципы нового подхода к оценкам инновационных проектов.

Во-первых, объектом оценки и расчетов является проект.

Как уже было сказано, в традиционной модели это детализация проекта и планирование работ. Входные данные при этом всегда строго задаются заказчиком. В инновационной модели входных данных для проекта нет. Оценку проекта производит не заказчик, а какая-либо заинтересованная сторона на основании описания проекта, сформулированного самим инноватором.

Во-вторых, в чем суть оценки и расчетов?

В первом случае это оценка соответствия объекта (проекта) заданным показателям, во втором - фиксация состояния проекта и прогноз его развития.

С системной точки зрения инновационный проект представляет собой набор функций и свойств атомов, молекул и проекта в целом. Каждая функция и свойство представляют собой вектор состояния и развития. В любой момент времени можно зафиксировать текущее состояние (числовая оценка) вектора и спрогнозировать направление и динамику развития. Причем планирование показателей и оценок векторов функций и свойств на длительную перспективу крайне затруднительно, так как по мере продвижения по циклу жизни на проект начинает действовать все большее число факторов, направление и характер воздействия которых предугадать заранее очень сложно.

Одним из центров кристаллизации проекта может быть (что правильно) инновационная идея. Однако в условиях перехода на новую модель экономики центрами кристаллизации могут быть инициативы любой заинтересованной стороны (инвестора, предприятия, государства, ученого сообщества и т.д.).

С системной точки зрения идея - это точка в информационном инновационном пространстве. Из нее начинают прорастать одномерные векторы состояний и оценок. Одни векторы имеют сильную взаимную зависимость, образуя двухмерные (плоскостные) состояния и оценки, другие могут образовывать и более сложные структуры. При этом большое значение имеет проектный этап цикла жизни. На ранних этапах состояния и оценки могут описываться в простейшей размерности (типа точки, «да-нет»). На последующих этапах размерность оценок может возрастать до двух и более измерений, причем независимо от этапа - имеет значение точка зрения и «удаленность» взгляда заинтересованной стороны. То есть возможно, что все детали проекта могут не интересовать оценивающую сторону и тогда оценка какой-то стороны проекта должна быть свернута в простейший показатель.

Из сказанного следует вывод, что объект оценки и расчетов (проект) имеет постоянно меняющиеся число и размерность параметров описания и оценки. Причем заранее задать или предугадать это число и размерность невозможно. Это связано с тем, что прорастание векторов идет по пути наименьшего сопро- 
тивления. Атомы инноватора прорастают (притягиваются) к атомам заинтересованных сторон исходя из декларированных ими намерений и подстраиваются под их формальные требования.

В общем случае число заинтересованных сторон может быть (должно быть) достаточно большим, поэтому и требования могут быть весьма разнообразными (несмотря на шаблонность конкурсной документации).

Необходимость постоянных оценок проекта не вызывает сомнений, так как на их основе заинтересованными сторонами принимаются все решения об объеме проделанной работы, о перспективности и эффективности проекта, о размере необходимых инвестиций, о рисках и неопределенностях и т.п.

Сложность оценок заключается в сложности структуры оцениваемого объекта, этапного проекта на цикл жизни и точки зрения оценщика.

Все сказанное приводит к предложению использовать при моделировании инновации и инновационной деятельности математический аппарат теории фракталов [8].

Фракталом может называться объект, обладающий, по крайней мере, одним из следующих свойств:

- нетривиальной структурой на всех масштабах; в этом отличие от регулярных фигур (таких как окружность, эллипс, график гладкой функции): если мы рассмотрим небольшой фрагмент регулярной фигуры в очень крупном масштабе, то он будет похож на фрагмент прямой, для фрактала увеличение масштаба не ведет к упрощению структуры, то есть на всех шкалах мы увидим одинаково сложную картину;

- самоподобностью или приближенно самоподобностью;

- дробной метрической размерностью или метрической размерностью, превосходящей топологическую.

Применительно к инновационному проекту это означает следующее. Под регулярностью структуры описания информационного объекта можно понимать такую степень детализации описания, когда оно может быть сведено к ответу на вопрос: да или нет. Но ни на каком уровне детализации никакая другая сторона, кроме инноватора, не может сформулировать такой вопрос, так как, кроме инноватора, никто не знает его идеи.

Поэтому структура даже атома инновации всегда остается нетривиальной. Более сложные структуры образуются из базовых элементов, но заранее предугадать узор проекта невозможно. В силу перечисленных выше причин меняется и размерность параметров оценки.

Можно определить инновационный проект как фрактал, который строится по принципу большого из малого по известным единообразным правилам.

Узор фрактала - это путь, по которому потенциал реализуется в атоме. За счет сил притяжения (намерений) атомы собираются в молекулы.

И в этом процессе нельзя простыми справками (вопросами и ответами) правильно оценить новую идею. Справки - это отражение опыта прошлых разработок, а инновация - новое предложение (изменение). Поэтому старые вопросы могут оказаться неуместными, создавать мощный барьер перед инновациями.

Как было сказано выше, для описания структуры инновации целесообразно использовать идеи ФСА и шкалирование информации на основе теории полезности.

В заключение следует отметить еще одно обстоятельство.

Для того чтобы в инновационном информационном пространстве возникали силы притяжения между атомами и молекулами, необходимо создавать и соблюдать условия конкуренции между всеми участниками внешней (инновационной) среды. Без конкуренции исчезает способность принятия риска. Это создает условия для развития и соединения безрисковых атомов и молекул, которые по определению являются обычными операциями и составляют основу традиционной, неинновационной экономики. В результате будут появляться и реализовываться неконкурентоспособные и неэффективные проекты, что сейчас и наблюдается повсеместно в инвестиционных структурах, созданных с государственным участием.

На основании изложенного можно сделать следующие выводы.

На инновацию не может быть написано техническое задание, так как инновационная экономика основывается не на спросе, а на достижениях науки и техники и инициативе творческих личностей, поэтому устройство инновационного проекта существенно отличается от традиционных проектов.

Инновационные проекты могут появляться только в условиях инновационного информационного пространства, состоящего из элементарных частиц, представляющих описание инновационных идей, намерений и состояний всех заинтересованных в инновациях сторон. Ключевыми заинтересованными сторонами для продвижения проекта являются инноватор, инвестор и потребитель инновации.

Хороший упакованный инновационный проект складывается из элементарных информационных частиц (атомов), а также необходимых и достаточных условий его поддержки и реализации. У каждой заинтересованной стороны свой атом. У всех атомов сходная структура, но атом инноватора имеет дополнительное описание (инновационной идеи). Атомы имеют внутренние (умение, желание, возможность) и внешние (новизна, реализуемость и выгодность) свойства. 
Необходимым условием для слияния атомов в молекулы является наличие сил притяжения этих атомов, которые проявляются через описание свойств ближней среды (предметная область, математические модели, информационные технологии).

Достаточное условие упакованности проекта - описание состояния внешней среды и самого инноватора (нормативная база, инновационная инфраструктура, предпринимательские способности инноватоpa).

Важнейшим вопросом в этой области является возможность оценки инновационного проекта на всех этапах проекта. Традиционные инвестиционные методики здесь неприменимы, так как оценке подвергаются будущие результаты без возможности подтверждения статистическими или рыночными данными.

Для оценки инновационных проектов целесообразно использовать теорию фракталов, позволяющих оценивать текущее состояние инновации и прогнозировать ее развитие по проектным этапам цикла жизни, а в качестве рабочего инструмента количественной оценки информации, имеющей качественный характер, - теорию полезности и методы функционально-стоимостного анализа.

Особое место в описании и оценке инновации и инновационного проекта занимают информационные технологии как инструмент формирования инновационного информационного пространства, без которого невозможны ни начало, ни развитие инновационной деятельности.

\section{Лuтература}

1. Семенов С.В. Инновации. Понятие и определения. URL: http://swsys-web.ru/innovation-concept-anddefinition.html (дата обращения: 10.02.2014).

2. Семенов С.В. Инновации. Инновационная деятельность. URL: http://swsys-web.ru/innovativeactivities.html (дата обращения: 10.02.2014).

3. Уёмов А.И. Системный подход и общая теория систем. М.: Мысль, 1978. 272 с.

4. Петров В. Основы ТРИЗ. Тель-Авив, 2010.

5. Котлер Ф. Основы маркетинга. М., 1990.

6. Грамп Е.А. Функционально-стоимостной анализ и его использование в промышленности зарубежных стран. М.: Информэлектро, 1971.

7. Тимоти Бадд. Объектно-ориентированное программирование в действии. СПб: Питер, 1997. 464 с.

8. Шредер М. Фракталы, хаос, степенные законы. Миниатюры из бесконечного рая. Ижевск: НИЦ «Регулярная и хаотическая динамика», 2001. 528 с. 